\title{
Uma investigação sobre a relação entre o projeto do produto e produção em uma montadora automotiva e fornecedores de motores que adotam a modularidade
}

\author{
Edilson Alves Rodrigues ${ }^{\mathrm{a}}$, José Antonio Carnevallib, \\ Paulo Augusto Cauchick Miguel ${ }^{\mathrm{c} *}$ \\ aedilson_rodrigues@hotmail.com, FAAP, Brasil \\ bjoseacarnevalli@uol.com.br, PUC-CAMPINAS, Brasil \\ c*cauchick@usp.br, USP, Brasil
}

\begin{abstract}
Resumo
A modularidade pode ser entendida como um conjunto de conceitos que leva a novas possibilidades de arquitetura de produto, bem como novas maneiras de organização da produção, possibilitando a transferência de atividades ao longo da cadeia de suprimentos automotiva. Nesse contexto, o objetivo deste trabalho é investigar o emprego da modularidade no projeto de produto e na produção, considerando uma situação de relação específica entre uma montadora de veículos comerciais e fornecedores de motores, no âmbito do consórcio modular. É também analisada a relação entre a modularidade no projeto de produto e na produção. Para a consecução desses objetivos é adotada a abordagem metodológica de estudo de caso. Os resultados apresentam algumas influências que ocorrem entre o projeto do produto e a produção e vice-versa, uma parte delas esperadas, reforçando o caráter confirmatório dos dados coletados. Constata-se que o conceito de modularidade pode ser aplicado ao processo produtivo sem necessariamente que o produto tenha sido concebido em módulos, ou seja, a partir de um produto existente é possivel montar seus componentes formando subconjuntos em uma lógica modular. Os dados confirmam ainda que a estrutura do produto de um veículo comercial favorece a sua divisão em módulos. Adicionalmente, a gestão da cadeia de suprimentos na montadora é simplificada em comparação ao sistema de produção convencional, pois parte das responsabilidades da montadora é transferida para os fornecedores.
\end{abstract}

Palavras-chave

Modularidade. Indústria automobilística. Projeto de produto. Desenvolvimento do produto.

\section{Introdução}

Em resposta à competição global, as empresas buscam aumentar a variedade e customizar seus produtos, bem como reduzir o seu tempo de vida, sem aumentar os custos de desenvolvimento e de produção (KOTLER, 2003). Para lidar com esse ambiente dinâmico, uma das estratégias adotadas pelas empresas é a introdução da modularidade. A modularidade tem como um dos principais objetivos a simplificação na gestão de um produto considerado complexo (BALDWIN; CLARK, 2000). Na verdade, a modularidade parte de um conjunto de conceitos que leva a novas formas de desenvolver os produtos, organizar a produção e transferir atividades que agregam valor para os fornecedores na cadeia produtiva (DORAN; HILL, 2008).

Em linhas gerais, a modularidade pode ser entendida como uma forma de concepção de um produto em subconjuntos menores, denominados módulos, mas que funcionam como um conjunto integrado (BALDWIN; CLARK, 1997), ou seja, um produto passa a ser constituído de módulos independentes, mas que funcionam de modo 
interdependente. lsso pressupõe que o projeto dos diversos módulos do produto pode ser realizado simultaneamente (SANCHEZ; MAHONEY, 1996). No âmbito da produção, significa que os módulos podem ser montados e testados em linhas separadas e só então enviados para a linha de montagem final do veículo na sequência requerida, ao invés da montagem de componentes avulsos, como no sistema convencional (SAKO; MURRAY, 2000; ULRICH, 1995). Do ponto de vista dos fornecedores de primeiro nível da cadeia de suprimentos, empregar a modularidade significa assumir um papel diferenciado do convencional, relativo às atividades de projeto de produto, montagem e entregas de subconjuntos (LUNG et al., 1999; MARX; ZILBOVICIUS; SALERNO, 1997).

A indústria automotiva utiliza a modularidade como forma de reduzir os custos de fabricação, como uma alternativa para lidar com a redução do ciclo de vida dos produtos (LAU ANTONIO; YAM; TANG, 2007), bem como para oferecer um número maior de modelos e variações no mercado, sem tornar o sistema demasiadamente complexo (PILLER; WARINGER, 1999). Aliado a isso, o tempo de desenvolvimento do produto tende a ser reduzido pela reutilização dos módulos e componentes entre os diferentes modelos de veículos (PERSSON; AHLSTROM, 2006; ARNHEITER; HARREN, 2005). Nesse sentido, a modularidade - que já era utilizada na linha de produção final havia muito tempo (BALDWIN; CLARK, 1997), como, por exemplo, para o projeto e montagem de motores e transmissões - passa então a ser utilizada como uma abordagem para o projeto do produto e para o processo de produção (SALERNO, 2001). Isso significa que, para explorar todos os benefícios da modularidade, é preciso conceber o produto em uma arquitetura modular e reproduzir essa estrutura no processo produtivo (FREDRIKSSON, 2006a). Um dos benefícios decorrentes da adoção da modularidade, no âmbito dos novos arranjos da cadeia produtiva automotiva, tem alterado as relações entre as montadoras e seus fornecedores, pois as decisões também envolvem a divisão de riscos e responsabilidades e investimentos entre as partes (SALERNO; CAMARGO; LEMOS, 2008).

Nesse sentido, este trabalho investiga o emprego da modularidade em um contexto específico na relação entre uma montadora de veículos comerciais e fornecedores de motores. Analisa-se a adoção da modularidade nas perspectivas do projeto do produto e da produção, além de verificar o relacionamento entre essas duas perspectivas, pois, apesar do avanço no uso da modularidade, poucos são os estudos empíricos que examinam esse relacionamento.

A seção seguinte deste trabalho apresenta a abordagem metodológica de pesquisa, caracterizando o ambiente investigado e os procedimentos para a coleta e análise dos dados. Em seguida, é apresentada uma breve revisão da literatura no que tange a alguns aspectos importantes associados à modularidade, sobretudo no projeto do produto e na produção modular. A quarta seção apresenta os resultados empíricos da análise entre a montadora automotiva e dois fornecedores. Por fim, a última seção apresenta os pontos conclusivos principais do presente trabalho.

\section{Abordagem metodológica de pesquisa}

Para atingir os objetivos deste trabalho, iniciou-se uma busca na literatura sobre o tema "modularidade", por meio de palavras-chave em base de dados relevantes. A partir dessa busca bibliográfica, levantaram-se aspectos relevantes relacionados ao tema investigado visando uma análise posterior mais aprofundada. A partir da identificação de alguns itens a serem investigados (apresentados no final do referencial teórico do trabalho), foi então feita a seleção das unidades de análise (casos), planejamento da coleta e da análise dos dados, visando, principalmente, analisar o alinhamento dos resultados com a literatura vigente disponível naquele momento.

Foi adotado o estudo de caso como abordagem metodológica de pesquisa na investigação, por considerá-lo mais adequado face aos seus objetivos, como estabelecido por Gil (2009). 0 autor supracitado considera como importante a natureza dos dados (qualitativos), o fenômeno, bem como o contexto da investigação. No presente trabalho, os dados a serem coletados e analisados são de natureza qualitativa, o fenômeno investigado é a adoção da modularidade no contexto de organizações do setor automotivo. A adoção do estudo de caso é relevante especialmente quando os limites entre o fenômeno investigado e o contexto não estão claramente definidos (YIN, 2005). Considera-se ainda que o trabalho apresenta caráter exploratório, principalmente pelo fato de que a relação entre a modularidade em projeto e em produção não é ainda plenamente estabelecida na literatura. De acordo com Cauchick Miguel (2007), utilizam-se estudos exploratórios quando a teoria não se encontra consolidada ou é emergente, ou seja, nesse trabalho pretende-se também identificar novas perspectivas a partir de estudos existentes.

Para a coleta de dados, foram realizadas entrevistas utilizando-se de roteiros com características semiestruturadas, conforme estabelecido por Marconi e Lakatos (2007). Elas foram feitas com engenheiros líderes e gerentes das áreas de engenharia do produto e de processo das empresas estudadas. 0 conteúdo das entrevistas foi gravado, transcrito e, posteriormente, enviado aos entrevistados para possíveis ajustes nas informações quanto à precisão e sigilo dos dados. 
Além delas, foram também utilizadas outras fontes de evidência. Por exemplo, após a entrevista na montadora foi possível participar de uma apresentação corporativa que forneceu elementos adicionais relacionados à produção e à parceria com os fornecedores. Outra fonte de evidência foi a observação direta por meio de visitas ao processo produtivo da montadora e dos fornecedores.

Para análise, o primeiro passo, a partir do conjunto de dados coletados, foi a sua organização, considerando as diferentes fontes de evidência. Em seguida, foi gerada uma narrativa geral dos casos, incluindo um conteúdo mais ligado ao objetivo do trabalho de pesquisa e, portanto, essencial para a análise. A partir dessa narrativa, buscou-se realizar comparações com a literatura, visando uma análise dos dados a partir de um referencial existente.

As empresas selecionadas para esta pesquisa foram escolhidas por utilizarem o conceito de modularidade (fenômeno) e por exercerem atividades associadas às perspectivas analisadas da modularidade (projeto de produto e produção) em suas operações no contexto da cadeia de suprimentos automotiva, premissas importantes para os objetivos deste trabalho. Outro aspecto que favoreceu a escolha da montadora analisada, embora de origem estrangeira, foi a sua autonomia no desenvolvimento de novos produtos no país.

A montadora de veículos comerciais opera o sistema conhecido como consórcio modular. Os dois fornecedores são posicionados no primeiro nível na cadeia de suprimentos, denominados de fornecedor A e B; se uniram formando uma joint venture para prestação de serviços de montagem do conjunto powertrain (motor, transmissão e sistema de resfriamento) nos veículos. Esses fornecedores projetam e produzem o módulo do motor, fornecendo-o em um sistema "caixa-preta".

\section{Base conceitual - principais conceitos associados à modularidade}

A modularidade consiste basicamente no processo de divisão de um produto em subconjuntos menores, chamados módulos, montados a partir de vários componentes (PERSSON, 2004). Com uma visão mais abrangente sobre o tema, Salerno, Camargo e Lemos (2008) propõem a modularidade como uma relação de serviços, pois além das atividades já praticadas e conhecidas, como projeto, produção e entrega de módulos pelo fornecedor, o uso da modularidade significa o compartilhamento de riscos com os fornecedores de primeiro nível. Esses fornecedores assumem a responsabilidade de alguns serviços, tais como assistência técnica, solução de problemas na linha de montagem, programação da produção e alterações no projeto do produto.

0 objetivo da modularidade é alcançar um produto em que os diferentes módulos sejam independentes uns dos outros, conectando-se por meio de interfaces especificadas e padronizadas, possibilitando alterações individuais sem que seja necessário interferir nos demais módulos (MIKKOLA, 2003; ULRICH, 1995). Para que isso seja alcançado, entretanto, alguns aspectos da modularidade que influenciam as decisões subsequentes ao processo de obtenção do produto devem ser respeitados, tais como (BALDWIN; CLARK, 1997): a) a arquitetura do produto, na qual especifica os módulos dos sistemas e suas funções; b) as interfaces, que são responsáveis pela ligação e interação entre os módulos; e c) a definição de padrões, cujo objetivo é verificar a conformidade do módulo. Do ponto de vista de engenharia, a modularidade apresenta 3 principais objetivos (BALDWIN; CLARK, 2000): a) uma forma de lidar com a complexidade do produto e processo, b) permitir a realização simultânea de atividades de projeto do produto e c) acomodar incertezas futuras associadas ao lançamento de novos produtos. Nesse sentido, a adoção da modularidade pode trazer vários benefícios para as empresas, como mostrado na Quadro 1.

Por outro lado, a modularidade também apresenta dificuldades na sua adoção, como mostrado na Quadro 2.

\subsection{Modularidade aplicada ao projeto do produto}

A modularidade aplicada ao projeto do produto visa a redução do tempo de desenvolvimento de produto por meio da execução de atividades em paralelo (BALDWIN; CLARK, 2000; ULRICH, 1995), ou seja, uma vez que as interfaces são especificadas, os módulos podem ser desenvolvidos independentemente e simultaneamente a outros módulos. As interfaces determinam como os módulos se conectam, se ajustam e se comunicam (CHEN; LIU, 2005; BALDWIN; CLARK, 1997). Dessa forma, a engenharia ganha mais liberdade para projetar e desenvolver os módulos, pois reduz a dependência direta de outras etapas do projeto do produto (ULRICH, 1995). Outro fator importante atribuído à independência entre os módulos é a intensidade de inovações no projeto, pois a engenharia pode criar e testar diferentes soluções nos seus próprios módulos, multiplicando as opções de produtos pelas relações entre os módulos (BALDWIN; CLARK, 2000).

\subsection{Modularidade aplicada à produção}

A produção eficiente de um portfólio diversificado de produtos é frequentemente atribuída à flexibilidade 
Quadro 1. Benefícios da modularidade identificados na literatura.

\begin{tabular}{|c|c|c|}
\hline Benefícios & Caracteristicas & Referências \\
\hline $\begin{array}{l}\text { Variedade de } \\
\text { produtos finais }\end{array}$ & $\begin{array}{l}\text { Ampla variedade de produtos finais a partir de } \\
\text { um número limitado de módulos, que podem ser } \\
\text { montados em diferentes configurações }\end{array}$ & $\begin{array}{l}\text { Doran e Hill (2008), Rozenfeld et al. (2006), Arnheiter } \\
\text { e Harren (2005), Chen e Liu (2005), Sanchez e Collins } \\
\text { (2001), Ulrich e Eppinger (2000), Baldwin e } \\
\text { Clark (1997) e Sanchez e Mahoney (1996) }\end{array}$ \\
\hline $\begin{array}{l}\text { Flexibilidade } \\
\text { da produção }\end{array}$ & $\begin{array}{l}\text { Os módulos podem ser pré-montados em linhas } \\
\text { separadas em vez de serem montados em } \\
\text { sequência na linha de montagem final }\end{array}$ & $\begin{array}{l}\text { Rozenfeld et al. (2006), Worren, Moore e Cardona } \\
\text { (2002), Sako e Murray (2000) e Baldwin e Clark (1997) }\end{array}$ \\
\hline $\begin{array}{l}\text { Redução do tempo } \\
\text { de desenvolvimento } \\
\text { do produto }\end{array}$ & $\begin{array}{l}\text { Os projetos dos módulos podem ser realizados quase } \\
\text { que de forma independente dos outros módulos, além de } \\
\text { utilizar soluções já desenvolvidas para outros componentes }\end{array}$ & $\begin{array}{l}\text { Rozenfeld et al. (2006), Baldwin e } \\
\quad \text { Clark (1997) e Ulrich (1995) }\end{array}$ \\
\hline Produtos customizados & Fornecer produtos conforme requisitos dos clientes & Arnheiter e Harren (2006), Persson $(2006,2004)$ \\
\hline $\begin{array}{l}\text { Economia } \\
\text { de escala }\end{array}$ & $\begin{array}{l}\text { Padronização de componentes } \\
\text { (reuso de componentes) }\end{array}$ & $\begin{array}{l}\text { Wu, De Matta e Lowe (2009), Eggen (2003), } \\
\text { Mikkola (2003) e Krishnan e Gupta (2001) }\end{array}$ \\
\hline Prestação de serviços & $\begin{array}{l}\text { Assistência técnica e solução de } \\
\text { problemas de qualidade na linha }\end{array}$ & Salerno, Camargo e Lemos (2008) \\
\hline $\begin{array}{c}\text { Compartilhamento } \\
\text { de riscos }\end{array}$ & $\begin{array}{l}\text { Os investimentos das montadoras são } \\
\text { compartilhados com os fornecedores de } 1^{\circ} \text { nível }\end{array}$ & $\begin{array}{c}\text { Salerno, Camargo e Lemos (2008) e Doran (2004), } \\
\text { Mikkola (2003) } \\
\end{array}$ \\
\hline $\begin{array}{c}\text { Redução de } \\
\text { custos de produção }\end{array}$ & $\begin{array}{l}\text { Reduzir custos de montagem e } \\
\text { investimentos em equipamentos }\end{array}$ & $\begin{array}{l}\text { Arnheiter e Harren (2005), Chen e Liu (2005), } \\
\text { Doran (2004) e Collins, Bechler e Pires (1997) }\end{array}$ \\
\hline $\begin{array}{c}\text { Possibilidade } \\
\text { de terceirização }\end{array}$ & $\begin{array}{l}0 \text { uso da arquitetura modular } \\
\text { favorece a terceirização }\end{array}$ & Doran e Hill (2008) e Takeishi e Fujimoto (2003) \\
\hline $\begin{array}{l}\text { Redução dos } \\
\text { níveis de estoque }\end{array}$ & $\begin{array}{l}\text { Os estoques são transferidos } \\
\text { para os fornecedores }\end{array}$ & Salerno et al. (2009) e Doran e Hill (2008) \\
\hline $\begin{array}{l}\text { Customização } \\
\text { em massa }\end{array}$ & $\begin{array}{l}\text { Ganhos de escala em } \\
\text { função da padronização }\end{array}$ & $\begin{array}{l}\text { Blees e Krause (2008), Arnheiter e Harren (2005) e } \\
\text { Worren, Moore e Cardona (2002) }\end{array}$ \\
\hline $\begin{array}{c}\text { Redução da } \\
\text { base de fornecedores }\end{array}$ & $\begin{array}{l}\text { Simplificação da rede de fornecimento pelo } \\
\text { agrupamento de componentes em módulos }\end{array}$ & $\begin{array}{c}\text { Arnheiter e Harren (2006), Baldwin e Clark (1997) e } \\
\text { Collins, Bechler e Pires (1997) }\end{array}$ \\
\hline $\begin{array}{l}\text { Redução } \\
\text { de custos }\end{array}$ & $\begin{array}{l}\text { Custos de desenvolvimento do produto e produção } \\
\text { (aumentar a produtividade) }\end{array}$ & $\begin{array}{l}\text { Arnheiter e Harren (2005), Doran (2004) e } \\
\text { Collins, Bechler e Pires (1997) }\end{array}$ \\
\hline $\begin{array}{c}\text { Redução da } \\
\text { base de fornecedores }\end{array}$ & $\begin{array}{c}\text { Simplificação da } \\
\text { cadeia de suprimentos }\end{array}$ & $\begin{array}{c}\text { Doran (2005), Baldwin e Clark (1997) e } \\
\text { Collins, Bechler e Pires (1997) }\end{array}$ \\
\hline Customização em massa & Ganhos de escala em função da padronização & Blees e Krause (2008) e Arnheiter e Harren (2005) \\
\hline
\end{tabular}

Quadro 2. Dificuldades na adoção da modularidade identificadas na literatura.

\begin{tabular}{|c|c|}
\hline Dificuldades & Referências \\
\hline Completo reprojeto do produto & Pandremenos et al. (2009), Takeishi e Fujimoto (2001) \\
\hline Esforço extra de projeto & Rozenfeld et al. (2006) \\
\hline Gerenciamento de tolerâncias (interfaces) & Pandremenos et al. (2009), Takeishi e Fujimoto (2001) \\
\hline Alto custo de manutenção/substituição & Pandremenos et al. (2009), Persson (2004) \\
\hline Perda de conhecimento específico & Pandremenos et al. (2009), Chesbrough e Teece (1996) \\
\hline Aumento da dependência do fornecedor & Frigant e lung (2002) \\
\hline
\end{tabular}

da manufatura (ULRICH, 1995), que é, em primeira instância, uma função da arquitetura do produto e, em segunda, da tecnologia utilizada na manufatura, dos centros de distribuição e da cadeia de suprimentos (RAMDAS, 2002). Em plantas convencionais, os componentes individuais e subconjuntos de um veículo são montados quase que individualmente no corpo do veículo na linha de montagem final (embora subconjuntos também sejam montados). A modularidade possibilita que os componentes e subconjuntos sejam montados em linhas de produção separadas, formando assim módulos, que são enviados para a linha de montagem final e instalados diretamente no veículo (SAKO; MURRAY, 1999; TAKEISHI; FUJIMOTO, 2001). Assim, há uma redução significativa na complexidade do processo de produção (ULRICH; TUNG, 1991; SAKO; MURRAY, 1999; TAKEISHI; FUJIMOTO, 2001) e, consequentemente, redução de custos de montagem (ULRICH; TUNG, 1991). Outro aspecto importante atribuído à modularidade na produção é a qualidade do produto final, pois os módulos facilitam a sua inspeção (TAKEISHI; FUJIMOTO, 2001), possibilitando identificar e resolver problemas o mais cedo possivel.

\subsection{A integração da manufatura e $o$ desenvolvimento de novos produtos}

A organização do processo produtivo é, geralmente, constituída de forma similar à estrutura do produto 
que as empresas projetam. Para um produto modular, em particular, à medida que as interfaces estão claramente definidas, o ideal é que o processo de produção seja desenvolvido seguindo a mesma estrutura do produto (FREDRIKSSON, 2006b). Para que isso ocorra, o PDP (processo de desenvolvimento de produto) necessita de informações da produção para realizar suas atividades, evitando, assim, problemas futuros na manufatura e montagem, investimentos desnecessários e atrasos no lançamento dos novos produtos (ROZENFELD et al., 2006). A manufatura, por sua vez, pode contribuir fornecendo informações sobre as restrições de capacidade produtiva, de processo e de tecnologias disponíveis (ROZENFELD et al., 2006). Dessa forma, os projetos do produto e do processo de produção devem caminhar em conjunto.

Com relação às formas de produção de um novo produto, estas podem ocorrer (ROZENFELD et al., 2006): a) em uma fábrica nova, onde a produção ocorrerá com máquinas, equipamentos, dispositivos e ferramentas novas; b) em instalações existentes, com parte ou todas as máquinas e equipamentos novos ou; c) em instalações e equipamentos existentes. No primeiro caso, como se trata de uma instalação nova, os recursos de produção são adquiridos conforme as necessidades. No segundo, é feita uma complementação dos recursos existentes, adquirindo assim máquinas, equipamentos, dispositivos e/ou ferramentas necessários. No último caso, é necessária uma avaliação com relação aos recursos necessários para a adequação do novo produto na produção. Nos dois últimos casos, como as instalações são existentes, muitas vezes é necessária uma adequação de layout.

\subsection{Consórcio modular e condomínio modular}

A reestruturação no setor automotivo resultou em formas alternativas de organização da cadeia produtiva: o consórcio modular e o condomínio industrial. Esses arranjos organizacionais apresentam como estratégia central a modularidade e a externalização (SACOMANO NETO; PIRES, 2007). Nesse sentido, a montadora passou a ter um relacionamento preferencial com os fornecedores de primeiro nível, aqueles que fornecem subconjuntos, sistemas ou módulos (SALERNO et al., 2001). No consórcio modular, os fornecedores de primeiro nível assumem a responsabilidade de montar os módulos e depois integrá-los ao veículo na linha de montagem final (PIRES; CARDOZA, 2007). Nesse caso, o fornecedor está instalado no mesmo prédio da montadora e caracteriza-se pelo fornecimento exclusivo. No caso do condomínio industrial, um grupo de fornecedores estratégicos de primeiro nível está alocado próximo à montadora, com o objetivo de abastecer a linha de montagem final do veículo numa forma just-in-sequence (PIRES; CARDOZA, 2007). Diferentemente do consórcio modular, no condomínio industrial a montadora é a responsável pela montagem do veículo na linha final.

\subsection{Sintese dos principais itens do trabalho de pesquisa}

Na revisão da literatura, identificaram-se alguns itens importantes relacionados ao projeto do produto e produção modular, apresentados na Quadro 3. Esses

Quadro 3. Itens identificados na literatura.

\begin{tabular}{|c|c|c|}
\hline Principais itens & Sintese & Referências \\
\hline $\begin{array}{c}\text { Arquitetura } \\
\text { modular do produto }\end{array}$ & $\begin{array}{c}\text { Compreende a disposição física dos componentes } \\
\text { do produto e como ocorrem as suas relações }\end{array}$ & $\begin{array}{c}\text { Fredriksson (2006a), Baldwin e Clark (2000) e } \\
\text { Ulrich (1995) }\end{array}$ \\
\hline $\begin{array}{l}\text { Padronização de componentes, } \\
\text { módulos e sistemas }\end{array}$ & $\begin{array}{l}\text { Permite o reuso de componentes, módulos e } \\
\text { sistemas }\end{array}$ & Krishnan e Gupta (2001) \\
\hline $\begin{array}{l}\text { Modularidade } \\
\text { na produção }\end{array}$ & $\begin{array}{c}\text { Possibilidade de montagem dos módulos em } \\
\text { linhas separadas e posteriormente enviados } \\
\text { para a linha de montagem final }\end{array}$ & $\begin{array}{c}\text { Worren, Moore e Cardona (2002), } \\
\text { Takeishi e Fujimoto (2001), Sako e Murray } \\
\text { (2000) e Baldwin e Clark (1997) }\end{array}$ \\
\hline $\begin{array}{l}\text { Flexibilidade } \\
\text { modular na produção }\end{array}$ & $\begin{array}{l}\text { Os módulos podem ser montados em } \\
\text { diferentes configurações do produto }\end{array}$ & $\begin{array}{c}\text { Doran e Hill (2008), Arnheiter e Harren (2005), } \\
\text { Chen e Liu (2005), Sanchez e Collins (2001), } \\
\text { Ulrich e Eppinger (2000), Baldwin e Clark } \\
\text { (1997) e Sanchez e Mahoney (1996) }\end{array}$ \\
\hline $\begin{array}{l}\text { A modularidade na produção } \\
\text { e sua influência no projeto do produto }\end{array}$ & $\begin{array}{l}\text { A organização do processo produtivo } \\
\text { existente em módulos pode influenciar } \\
\text { o projeto do produto modular }\end{array}$ & $\begin{array}{c}\text { Fredriksson (2006a), Rozenfeld et al. (2006) e } \\
\text { Takeishi e Fujimoto (2001) }\end{array}$ \\
\hline $\begin{array}{l}0 \text { desenvolvimento do produto modular } \\
\text { e sua influência na linha de produção }\end{array}$ & $\begin{array}{l}\text { A estrutura do produto modular é utilizada para } \\
\text { definir a estrutura do processo produtivo }\end{array}$ & $\begin{array}{c}\text { Fredriksson (2006a); Rozenfeld et al. (2006) e } \\
\text { Takeishi e Fujimoto (2001) }\end{array}$ \\
\hline $\begin{array}{l}\text { Gestão do produto } \\
\text { e produção modular }\end{array}$ & $\begin{array}{l}\text { Redução da complexidade de gestão } \\
\text { da cadeia de suprimentos }\end{array}$ & Baldwin e Clark (2000) e Ulrich (1995) \\
\hline $\begin{array}{l}\text { Terceirização de atividades de projeto } \\
\text { do produto e produção modular }\end{array}$ & $\begin{array}{c}\text { A subcontratação de atividades de projeto do } \\
\text { produto e produção serve como alternativa } \\
\text { para a redução de custos }\end{array}$ & $\begin{array}{l}\text { Doran (2005), Pires (1998) e } \\
\text { Collins, Bechler e Pires (1997) }\end{array}$ \\
\hline
\end{tabular}


itens foram selecionados pela sua importância em relação aos objetivos do trabalho.

Os itens da Quadro 3 são analisados então em três contextos importantes (desenvolvimento do produto modular, consórcio modular na produção e relação entre a montadora e fornecedores), cujos resultados são apresentados a seguir.

\section{Resultados e discussão}

Nesta seção são apresentados os resultados dos casos por meio de uma narrativa geral, buscando-se uma comparação com a literatura para realizar a análise dos dados coletados.

\subsection{Contexto e resultados da coleta dos dados - montadora}

A montadora de veículos comerciais investigada, segundo dados da Anfavea (2009), ocupa uma posição de destaque na fabricação de caminhões e chassis de ônibus, marcada pela forma em que efetua a gestão da produção conhecida como consórcio modular. Nesse sistema de arranjo organizacional, sete fornecedores de primeiro nível fornecem e montam os módulos nos veículos na linha de produção em sete módulos (chassis; eixos e suspensão; rodas e pneus; motores, transmissões e sistema de resfriamento; cabines; pintura e acabamento interno da cabine) sob a supervisão e acompanhamento da montadora. Dessa forma, a montadora pode se concentrar no projeto do produto, em serviços ao cliente, marketing e vendas, introduzindo veículos mais apropriados para as necessidades do mercado. Este é um benefício da modularidade já apontado há algum tempo pela literatura (COLLINS; BECHLER; PIRES, 1997; MARX; ZILBOVICIUS; SALERNO, 1997).

\subsubsection{Desenvolvimento do produto modular}

A empresa possui um centro de pesquisa e desenvolvimento (P\&D) no Brasil para projeto e lançamento de caminhões e chassis de ônibus. Essa competência no desenvolvimento do produto representa um aspecto econômico importante, pois gera a necessidade de funcionários mais qualificados (SALERNO et al., 2001). Outro aspecto relevante é a possibilidade de realização de projetos e coprojetos (co-design) com os fornecedores, o que de certa forma beneficia os fornecedores instalados no país (SALERNO et al., 2009). Esses dois aspectos anteriores, destacados pela literatura, estão presentes na empresa investigada. 0 projeto do veículo é totalmente realizado pela montadora no que diz respeito aos requisitos veiculares e conta com a parceria de seus fornecedores para o desenvolvimento dos módulos como, por exemplo, cabines, motores e transmissões. A engenharia da montadora em questão é dividida em especialidades: powertrain, elétrica, cabine e chassi, sendo o PDP dividido em um modelo de referência de estágios e gates; esta divisão é relativamente comum no setor automotivo.

A divisão dos produtos "caminhão" e "chassi de ônibus" em módulos é facilitada devido ao fato de que as características de arquitetura desses produtos são mais apropriadas. No caso de um caminhão, por exemplo, a separação da cabine e do chassi é facilitada para a composição de diferentes modelos. Além disso, o chassi serve como base para o desenvolvimento da lógica modular, corroborado por outras fontes bibliográficas (CAUCHICK MIGUEL; PIRES, 2006; MARX; ZILBOVICIUS; SALERNO, 1997). Essa situação é diferenciada quando comparada a um veículo de passeio, considerado um produto complexo (PERSSON; AHLSTROM, 2006), mais voltado para uma arquitetura integrada. Na coleta de dados, identificou-se que a divisão inicial do produto em módulos foi realizada empiricamente, quando as engenharias da montadora e de seus fornecedores definiram, na linha de montagem, as interfaces de cada módulo. A partir da interdependência de cada módulo, visando seu funcionamento adequado, foi definida para qual fornecedor ficaria a responsabilidade de montar os acessórios (mangueiras, conexões, etc.). Ou seja, a montadora partiu de um produto existente para a criação do conceito de produto modular, sendo este um possível indicativo de que o projeto modular e a produção modular não são necessariamente (e obrigatoriamente) dependentes. 0 entrevistado mencionou terem ocorrido várias alterações no projeto, visando adaptá-lo modularmente, mas não as identificou no momento da entrevista.

Em função de um dos últimos lançamentos da empresa foi necessário o apoio da engenharia da matriz no desenvolvimento da cabine o que, aparentemente, revela uma possível contradição dos dados coletados em relação à autonomia da planta em P\&D. Para esse lançamento, foi necessário o desenvolvimento de uma nova cabine, motores e suspensão. 0 projeto nasceu com a participação e envolvimento de diversas áreas da montadora (logística, qualidade, manufatura e produto). Com o desenvolvimento do novo produto foi construída a segunda linha de armação de cabines. No mesmo período também foi criada uma planta piloto, cujo objetivo é simular o processo de montagem, identificando possíveis interferências de um novo desenvolvimento de produto. 


\subsubsection{Relação entre a montadora e fornecedores}

A coleta de dados identificou alguns elementos importantes que dizem respeito à organização da produção na montadora investigada, no que tange à relação com os fornecedores. Esses elementos são apresentados a seguir e comparados com a literatura quando pertinente:

- A montadora é responsável pela seleção, desenvolvimento e pagamento dos fornecedores de segundo e terceiro níveis. No entanto, atribui aos fornecedores de primeiro nível lá instalados a responsabilidade pela programação de pedidos, o controle da qualidade e dos prazos de entrega dos componentes, como destacam Rachid et al. (2006). Dessa maneira, o monitoramento da montadora com relação a esses pedidos é efetuada de forma rigorosa a fim de não gerar alto inventário físico. 0 alto poder de negociação da montadora pode ser considerado como justificativa para ainda permanecer no controle de aquisição de componentes, como explanado por Sacomano Neto e Pires (2007);

- 0 fornecedor de primeiro nível possui infraestrutura e os recursos necessários para a montagem dos módulos nos veículos na planta do consórcio modular. Essas instalações são caracterizadas por dedicação exclusiva à montadora. Esta pode ser considerada uma das desvantagens do consórcio modular para o fornecedor de primeiro nível, pois aumenta a interdependência entre as empresas em relação à demanda de produção da montadora. Por outro lado, estabelece uma relação de médio-longo prazo, pelo menos enquanto permanecer a vigência contratual entre as partes;

- Os créditos referentes aos serviços prestados acontecem em duas etapas, que são determinadas por pontos de inspeções na produção. 0 fornecedor recebe uma parte do pagamento quando é completada a montagem do veículo e o restante quando o veículo é aprovado nos testes finais de qualidade. Esse fato não se alterou desde o início do consórcio modular. Nesse tipo de abordagem, o pagamento é realizado conforme a produção (SALERNO; CAMARGO; LEMOS, 2008), o que é criticado por Rachid et al. (2006), que alegam que isso cria uma pressão para aumentar a produtividade;

- Os novos investimentos são negociados entre os fornecedores de primeiro nível e a montadora para definição da participação de cada um. 0 mais comum é o fornecedor investir e a montadora amortizar na quantidade de veículos produzidos, ou seja, uma parte fixa devido à amortização do capital investido e a outra variável de acordo com a quantidade de veículos produzidos e aprovados na linha de montagem final em um dado período.
Tal prática traz para a montadora benefícios, pois parte do pagamento do investimento é realizada com a própria produção de veículos;

- Os fornecedores de primeiro nível são responsáveis pelos módulos fornecidos e pela qualidade dos serviços prestados na linha de montagem. Este é mais um fato confirmatório, já destacado na fundamentação teórica, ou seja, o relacionamento entre a montadora e o fornecedor vai além de projeto e montagem, assumindo também a responsabilidade de alguns serviços, como é destacado mais recentemente por Salerno, Camargo e Lemos (2008);

- Na produção são realizadas reuniões diárias entre representantes da montadora e fornecedores, cujo objetivo é rever o plano mestre de programação. Assim, se há algum problema de qualidade ou abastecimento é tomada a decisão junto com a montadora para alteração do planejamento. Essa prática organizacional é mostrada como benefício de parceria gerada pela modularidade (ARNHEITER; HARREN, 2005);

- A programação da produção é do tipo taylor made, no qual o veículo vendido na concessionária envia o pedido direto para a linha de produção. Na literatura mais antiga (COLLINS; BECHLER; PIRES, 1997) é citado como make-to-order. Embora esse elemento não tenha sido investigado em profundidade, acredita-se que tanto o produto quanto a produção organizados modularmente favorecem esta prática da empresa no âmbito da customização de produto (MONDRAGON; MONDRAGON; MILLER, 2006);

- No caso do desenvolvimento de novos produtos, a montadora é responsável pelo desenvolvimento do projeto do veículo. Apesar de o entrevistado não ter indicado o envolvimento dos fornecedores no desenvolvimento do módulo, o cruzamento dos dados com os resultados dos fornecedores A e B (apresentados a seguir) indica a existência dessa parceria.

\subsection{Contexto e resultados da coleta dos dados - fornecedores $A$ e $B$}

A coleta de dados também envolveu fornecedores de primeiro nível fabricantes de motores, buscando compreender como foram tomadas as decisões de adequação dos produtos em relação ao novo arranjo produtivo modular e como ocorreu o desenvolvimento de novos motores para a montadora. Os fornecedores A e B são empresas multinacionais que adotam o conceito de modularidade.

No consórcio modular, os fornecedores A e B operam através de uma joint venture - parceria - formada entre as empresas para prestação de serviços de montagem do veículo na planta da montadora investigada. 
Vale destacar que, fora do consórcio modular, as empresas são concorrentes no segmento de motores de média potência. A Figura 1 mostra o recorte das atividades exercidas pelos fornecedores A e B na cadeia de suprimentos automotivos. 0 primeiro bloco de linhas tracejadas mostra as atividades dos fornecedores A e B individualmente, ou seja, cada um em sua planta; compreendem as etapas de projeto do motor, fabricação ou aquisição de componentes e pré-montagem do motor. 0 segundo bloco de linhas tracejadas representa as atividades na joint venture - efetuadas na planta da montadora. Nesse bloco, a transmissão e o sistema de arrefecimento são agregados ao motor, formando o conjunto powertrain. Em seguida, esse módulo é montado no chassi do veículo.

\subsubsection{Projeto do produto e aspectos de montagem no consórcio modular}

A redefinição das responsabilidades e a transferência de atividades de projeto do produto para os fornecedores de primeiro nível foram baseadas no funcionamento do módulo. Por exemplo, se para o funcionamento do motor é necessário um radiador, então todos os componentes e subconjuntos requeridos para interligar esses componentes ficaram sob a responsabilidade de montagem do fornecedor do módulo do motor.

Os fornecedores A e B participaram da criação do consórcio modular auxiliando nas definições organizacionais de layout, logística e processo de produção. Naquela época, cada fornecedor de primeiro nível definiu seu processo de montagem e os recursos necessários para efetuar as suas atividades.

Os espaços físicos ocupados pelos fornecedores de primeiro nível foram definidos próximos à linha de montagem final e dispostos em uma sequência lógica de montagem do veículo. 0 arranjo físico dos fornecedores na linha de montagem final indica que a organização da produção apresenta certa relação com a estrutura do produto. Em função da arquitetura do produto e da concepção de modularidade utilizadas na nova planta, algumas alterações na sequência de montagem do veículo e do layout do produto foram necessárias quando comparadas com a forma convencional de produção. Essas alterações visaram melhorar a forma de trabalho buscando também lidar com uma montagem diferenciada quando comparada com a tradicional, pois esse sistema de produção envolve um módulo constituído pelo motor e seus acessórios. Essa constatação é coerente com estudo mais antigos (MARX; ZILBOVICIUS; SALERNO, 1997). Contraditoriamente, no caso do projeto do carro smart foi necessário reprojetar por completo o produto e o processo de fabricação (HOEK; WEKEN, 1998), o que não ocorreu na presente investigação.

\subsubsection{O desenvolvimento de produto nos fornecedores}

Segundo os dados coletados, o processo de desenvolvimento de novos produtos nos fornecedores A e B pode acontecer de 3 formas: a) para incorporar novas tecnologias, como no caso do motor eletrônico (i.e. motores com módulo de controle eletrônico), que substitui o motor mecânico em várias aplicações; b) por uma necessidade de atendimento do cliente e mercado ou; c) para atendimento a novas legislações. No caso de motores, as solicitações com relação às emissões de gases são cada vez mais rigorosas e constantes.

Ainda segundo os dados coletados, os projetos de desenvolvimento do produto dos fornecedores A e B são classificados em projetos de motores "básico" e de "aplicação":

- Motor básico: consiste na criação de um novo motor. Esses projetos podem ser realizados sem a existência de um cliente específico, vislumbrando um nicho de mercado ainda não explorado, por exemplo;

- Aplicação: relacionados aos componentes e sistemas de interface do motor com outros módulos e chassi

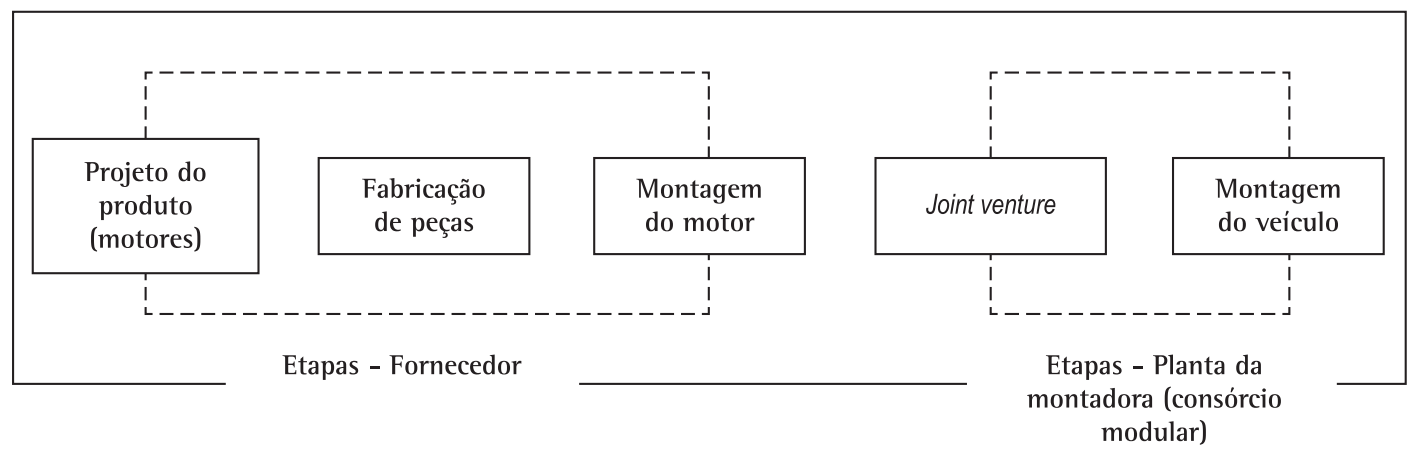

Figura 1. Diagrama representativo do fornecimento de motores (fornecedores A e B). 
do veículo, desenvolvidos para aplicação de um motor básico em um veículo de um cliente específico. Ocorrem à medida que existe um motor básico desenvolvido ou ao menos que o projeto esteja num estágio avançado de desenvolvimento. No caso da aplicação de um motor básico ainda em desenvolvimento, este deve ter sido aprovado, pelo menos, em testes de confiabilidade e durabilidade.

0 desenvolvimento do projeto de um motor básico ocorre por meio de um conjunto de requisitos da montadora, mas é totalmente desenvolvido pelos fornecedores num sistema do tipo "caixa-preta". Nesse tipo de desenvolvimento, o OEM (original equipment manufacturer) determina as especificações de desempenho e a concepção e detalhamento fica por conta do fornecedor. No caso do projeto de aplicação, este pode ser considerado como um coprojeto, pois o fornecedor e a montadora juntam esforços para definição dos requisitos de projeto. Essa prática é destacada por Mikkola (2003).

Durante o desenvolvimento do projeto de aplicação, a montadora tem como responsabilidade informar os requisitos veiculares, o cronograma de desenvolvimento do produto e a disponibilizar um caminhão para testes que será utilizado durante o desenvolvimento do novo produto. Para o desenvolvimento de motores e acessórios, o fornecedor B procura manter as interfaces padronizadas, mesmo que os motores básicos sejam diferenciados, até em cilindradas diferentes. Esse aspecto reduz significativamente os investimentos em ferramentas e as variações nos processos de produção. Sendo assim, tende a aumentar a qualidade do produto à medida que reduz os riscos de erros de montagens. Com relação aos componentes ou subconjuntos externos do motor, Arnheiter e Harren (2006) destacam, por exemplo, que os serviços para substituição de filtros de óleo e demais componentes externos do motor tornam-se mais rápidos à medida que as interfaces são padronizadas.

A padronização no desenvolvimento de novos produtos, como, por exemplo, o desenvolvimento do módulo de controle eletrônico para utilização em nível global e a padronização dos pistões dos motores, possibilita um aproveitamento do desenvolvimento do produto e do processo. Consequentemente, gera uma redução na variedade de peças, inventário físico e logística, como aponta a literatura (MIKKOLA, 2003; SANCHEZ, 2002).

Em um dos últimos lançamentos de veículo comercial da montadora investigada, o fornecedor A desenvolveu duas linhas de motores. A cada nova legislação que deve ser atendida, surge a necessidade de acrescentar componentes para a adequação do módulo motor. Nesses casos, o projeto do produto é realizado com alto grau de incerteza inicial, e, à medida que as informações mais precisas são definidas, ocorrem modificações nas fases seguintes do desenvolvimento.

Com o surgimento de novas tecnologias, como, por exemplo, o módulo de controle eletrônico do motor, houve alterações no projeto de aplicação para um novo caminhão da montadora. Essa alteração permitiu maior customização do produto no atendimento das necessidades do consumidor final; a customização pode ser entendida como um benefício da modularidade (DANESE; ROMANO, 2004; MONDRAGON; MONDRAGON; MILLER, 2006).

Com relação à transferência de conhecimento e know how da montadora para o fornecedor B, no que diz respeito à tecnologia de motores, isso praticamente não acontece, pois a tecnologia core é do fornecedor B. Por outro lado, apesar de o fornecedor aprender muito sobre o veículo, isto é um risco pois, de acordo com Mondragon, Mondragon e Miller (2006), a contínua transferência de atividades para seus fornecedores têm feito algumas montadoras automotivas perder suas competências, especialmente em subconjuntos.

\subsubsection{Consórcio modular na produção}

As engenharias de manufatura dos fornecedores A e B participam desde o início do desenvolvimento dos novos produtos com um time multifuncional. Primeiramente, esse time atua na concepção do projeto do motor e, num segundo estágio, interage com a joint venture no desenvolvimento do processo de montagem do veículo. Segundo as entrevistas e observações in loco, as operações efetuadas na linha de montagem do veículo são relativamente simples, geralmente executadas com o auxílio de pontes rolantes, balancins, dispositivos de içamentos e apertadeiras eletrônicas manuseadas pelos operadores, flexiveis para montagem de diferentes motores em qualquer família de produtos. A redução do manuseio de componentes na linha de montagem final e a transferência de montagem dos módulos para outras linhas de produção são destacadas como um dos benefícios do uso da modularidade (FREDRIKSSON, 2006b; HOEK; WEKEN, 1998).

Uma das dificuldades encontradas pelos fornecedores A e B foi a comunização das interfaces entre os seus motores para a montagem do módulo na linha de montagem final. Como os motores têm projetos diferenciados, isso faz com que sejam mantidos em estoque os diversos componentes utilizados nas interfaces de cada produto.

Para o fornecedor $A$, com relação às interfaces mecânicas, os motores tinham inclinações diferentes quando comparados aos modelos de cabine antiga e ao atual. Assim, foram necessárias várias alterações, 
pois diversos acessórios também foram modificados. Entretanto, segundo o entrevistado, essas alterações não afetaram a linha de montagem final da joint venture, o que parece indicar, nesse caso do módulo de motores, que existe certa independência entre modularidade do produto e produção.

No processo produtivo, as alterações ocorreram, basicamente, em função do uso de novas tecnologias, ou seja, a parte eletrônica mudou, pois a interface eletrônica, que antes não existia, passou a ser feita com os chicotes elétricos, além da integração de um módulo de controle eletrônico e vários sensores.

\subsubsection{Cooperação com os fornecedores}

0 PDP do fornecedor B é cada vez mais realizado em conjunto com seus fornecedores que atuam desde o início do projeto até o momento de realização de testes funcionais no cliente final (OEM). Segundo os dados coletados, geralmente, a parceria com os fornecedores para o desenvolvimento de novos produtos acontece em um sistema de coprojeto. A parceria com alguns fornecedores-chave tem como objetivo principal a redução de custos por meio da transferência de atividades na cadeia de suprimentos como, por exemplo, a montagem de anéis no pistão do motor. Segundo Rozenfeld et al. (2006), nesse caso os fornecedores são considerados membros do time de desenvolvimento e a terceirização que ocorre no fornecedor B vai ao encontro do estudo de Doran (2004), que afirma que os fornecedores de primeiro nivel tendem a se concentrar em atividades de maior valor agregado e transferir as de menor valor para os fornecedores abaixo na cadeia de suprimentos, como destaca estudos mais recentes no país (PRIET0; CAUCHICK MIGUEL, 2011).

\section{Conclusões}

Em linhas gerais, a partir dos dados de campo, constata-se que o conceito de modularidade pode ser aplicado ao processo produtivo sem necessariamente que o produto tenha sido concebido em módulos, ou seja, a partir de um produto existente é possível montar seus componentes formando subconjuntos em uma lógica modular. Certamente, isto não é possivel para todo o veículo (particularmente no caso investigado de caminhões e chassis de ônibus), principalmente se este não foi projetado segundo o conceito de arquitetura modular. No entanto, os dados coletados mostraram que o uso da modularidade, na montadora investigada, nasceu na produção, baseado em decisões empiricas de montagem e funcionamento do módulo.
Por outro lado, quando um produto é concebido em módulos, a decorrência, em geral, é que o processo produtivo seja organizado de acordo com a estrutura do produto. Nesse sentido, a modularidade no projeto do produto pode levar à modularidade no processo produtivo. Entretanto, não é possível ser conclusivo quanto a esta afirmativa na presente investigação. Especificamente no presente trabalho, tanto a literatura quanto os dados coletados (entrevistas e observações in loco) evidenciaram que a estrutura do produto de um veículo comercial favorece a divisão do produto em módulos, uma vez que o chassi constitui uma estrutura técnica adequada como base para o desenvolvimento da lógica modular.

Com relação à produção, a aplicação do conceito de modularidade na linha de montagem final parece tornar o processo de produção mais simplificado, pois o número total de componentes e subconjuntos manuseados é reduzido. Além disso, os módulos são agregados ao veículo por meio de interfaces padronizadas. Pode-se, então, concluir que alguns dos fatores que contribuem para a simplificação do processo de produção correspondem ao uso de ferramentas padronizadas, uma vez que componentes entre diferentes linhas de produtos são padronizados e há utilização de módulos comuns entre diferentes produtos.

No consórcio modular, como os módulos são fornecidos montados, a linha de montagem final pode ser significativamente reduzida em termos de extensão, pois fará apenas a integração destes. Como consequência, há indicações conclusivas de que o aporte de recursos da montadora na linha de montagem em máquinas, equipamentos, dispositivos e ferramentas é reduzido, pois o investimento deve ser menor. No entanto, não foi possível apurar esse montante na presente investigação, pois dados de custos não foram disponibilizados.

Com relação à organização do sistema produtivo existente (linha de montagem final), esta pode influenciar o desenvolvimento de novos produtos, nos seguintes aspectos: a) em função das máquinas, equipamentos, dispositivos, fluxo de movimentação de materiais, layout, entre outros recursos existentes, torna-se necessário promover alterações no desenho do produto para adequá-lo ao processo e; b) adequação do produto, no sentido de alterar o posicionamento de mangueiras, de chicotes elétricos, entre outros componentes ou subconjuntos para facilitar ou permitir a operação de montagem.

Por outro lado, o desenvolvimento do produto modular também pode influenciar o sistema produtivo (linha de montagem final). lsso acontece, principalmente, quando o processo produtivo não tem capacidade tecnológica de produção do produto 
desenvolvido. Constata então que a estrutura do produto influencia na definição de recursos necessários para a produção, que são determinados de acordo com os métodos de montagem, a configuração dos módulos, que apresenta características diferentes de montagem quando comparada a um produto integrado. Essas constatações são relativamente esperadas, fazendo com que esta investigação seja de caráter confirmatório. Mesmo com essa ressalva, o diferencial do trabalho vai além da verificação do emprego da modularidade no projeto de produto e na produção, resultando em uma análise, mesmo em nível confirmatório, da relação entre essas perspectivas no contexto modular.

Ainda em relação ao contexto modular, destaca-se ainda que a complexidade da gestão da cadeia de suprimentos para a montadora é simplificada quando comparada com o sistema de produção tradicional, pois as responsabilidades são transferidas aos fornecedores de primeiro nível na cadeia de suprimentos, que ficam encarregados de desenvolver os fornecedores nos elos subsequentes da cadeia, de negociar, coordenar a qualidade e a logística dos materiais. Por outro lado, acredita-se que a complexidade na gestão de contratos com os fornecedores de primeiro nível aumenta, pois os contratos tendem a ser de longo prazo e exclusivos e, em alguns casos, incluindo os projetos futuros de produtos.

De forma geral e restrita ao trabalho realizado, por mais que o produto e o processo produtivo sejam planejados, o produto modular sempre estará sujeito a alterações durante sua vida útil. As alterações podem surgir em decorrência de otimizações do processo produtivo e dos produtos existentes ou com a introdução de novos produtos, adaptações a novas legislações, detecção de falhas, redução de custos, entre outras necessidades. Dessa forma, a análise de necessidade de adaptação do produto e/ou do processo acontece de forma contínua durante sua vida útil, ressaltando que esse aspecto deve ser investigado em maior profundidade em estudos futuros.

\section{Referências}

ARNHEITER, E. D.; HARREN, H. A typology to unleash the potential of modularity. Journal of Manufacturing Technology Management, v. 16, n. 7, p. 699-711, 2005. http://dx.doi.org/10.1108/17410380510619923

ARNHEITER, E. D.; HARREN, H. Quality management in a modular world. The TQM Magazine, v. 18, n. 1, p. 8796,2006.http://dx.doi.org/10.1108/09544780610637712

ASSOCIAÇÃO NACIONAL DOS FABRICANTES DE VEÍCULOS AUTOMOTORES - ANFAVEA. Anuário da Indústria Automobilística Brasileira. São Paulo: AutoData, 2009. Brazil Automotive Guide.
BALDWIN, C. Y.; CLARK, K. B. Managing in an age of modularity. Harvard Business Review, v. 75, n. 5 , p. 84-93, 1997.

BALDWIN, C. Y., CLARK, K. B. Design Rules: The Power of Modularity. Cambridge: MIT Press, 2000.

BLEES, C.; KRAUSE, D. On the development of modular product structure: a differentiated approach. In: INTERNATIONAL DESIGN CONFERENCE, 2008, Dubronik. Proceedings... Dubronik, Croatia, 2008.

CAUCHICK MIGUEL, P. A. Estudo de caso na engenharia de produção: estruturação e recomendações para sua condução. Produção, v. 17, n. 1, p. 216-229, 2007.

CAUCHICK MIGUEL, P. A.; PIRES, S. R. 1. A case study on modularity in product development and production within the auto industry. International Journal of Automotive Technology and Management, v. 6 , n. 3, p. 315-330, 2006. http://dx.doi.org/10.1504/ IJATM.2006.012123

CHEN, K. M.; LIU, R. J. Interface strategies in modular product innovation. Technovation, v. 25, p. 771-782, 2005 http://dx.doi.org/10.1016/j.technovation.2004.01.013

CHESBROUGH, H. W.; TEECE, D. J. When is virtual virtuous? Organizing for innovation. Harvard Business Review, n. 74, p. 65-73, 1996.

COLLINS, R.; BECHLER, K.; PIRES, S. Outsourcing in the automotive industry: from JIT to modular consortia. European Management Journal, v. 15, n. 5, p. 498-508, 1997. http://dx.doi.org/10.1016/S02632373(97)00030-3

DANESE, P.; ROMANO, P. Improving inter-functional coordination to face high product variety and frequent modifications. International Journal of Operations \& Production Management, v. 24, n. 9, p. 863-885, 2004. http://dx.doi.org/10.1108/01443570410552090

DORAN, D. Supplying on a modular basis: an examination of strategic issues. International Journal of Physical Distribution \& Logistics Management, v. 35, n. 9, p. $654-663,2005$. http://dx.doi. org/10.1108/09600030510632023

DORAN, D. Supply Chain management. Rethinking the supply chain: an automotive perspective. Supply Chain Management: an International Journal, v. 9, n. 1, p. 102-109, 2004. http://dx.doi. org/10.1108/13598540410517610

DORAN, D.; HILL, A. A review of modular strategies and architecture within manufacturing operations. Journal of Automobile Engineering, v. 223, p. 65-75, 2008. http:// dx.doi.org/10.1243/09544070JAUT0822

FREDRIKSSON, P. Cooperation and conflict in modular production and supplier parks: the case of Volvo cars modular assembly system. International Journal of Automotive Technology and Management, v. 6, n. 3, p. 298-314, 2006a. http://dx.doi.org/10.1504/ IJATM.2006.012122

FREDRIKSSON, P. Mechanisms and rationales for coordination of a modular assembly system: the case of Volvo cars. International Journal of Operations \& Production Management, v. 26, n. 4, p. 350-370, 2006b. http:// dx.doi.org/10.1108/01443570610650530

FRIGANT, V.; LUNG, Y. Geographical proximity and supplying relationships in modular production. International Journal of Urban and Regional Research, v. 26, n. 4, p. 742-755, 2002. http://dx.doi.org/10.1111/14682427.00415 
GlL, A. C. Estudo de caso: fundamentação científica, subsídios para coleta e análise de dados, como redigir o relatório. São Paulo: Editora Atlas, 2009.

HOEK, R. 1. V.; WEKEN, H. A. M. The impact of modular production on the dynamics of supply chains. The International Journal of Logistics Management, v. 9, n. 2, p. 35-50, 1998. http://dx.doi. org/10.1108/09574099810805825

KOTLER, P. A framework for marketing management. 2. ed. New Jersew: Pearson Education International, 2003.

KRISHNAN, V.; GUPTA, S. Appropriateness and impact of platform-based product development. Management Science, v. 47, n. 1, p. 52-68, 2001. http://dx.doi. org/10.1287/mnsc.47.1.52.10665

LAU ANTONIO, K. W.; YAM, R. C. M; TANG, E. The impacts of product modularity on competitive capabilities and performance: an empirical study. International Journal Production Economics, v. 105, p. 1-20, 2007. http:// dx.doi.org/10.1016/j.ijpe.2006.02.002

LUNG, Y. et al. Coping with variety: flexible productive systems for product variety in the auto industry. Aldershot: Ashgate, 1999.

MARCONI, M. D. A.; LAKATOS, E. M. Técnicas de pesquisa: planejamento e execução de pesquisas, amostragens e técnicas de pesquisas, elaboração, análise e interpretação de dados. 7. ed. São Paulo: Atlas, 2008.

MARX, R.; ZILBOVICIUS, M.; SALERNO, M. S. The modular consortium in a new VW truck plant in Brazil: new forms of assembler and supplier relationship. Integrated Manufacturing Systems, v. 8, n. 5, p. 292-298, 1997. http://dx.doi.org/10.1108/09576069710179742

MIKKOLA, J. H. Modularity, component outsourcing, and inter-firm learning. $R \& D$ Management, v. 33, n. 4 , p. 449-454, 2003. http://dx.doi.org/10.1111/14679310.00309

MONDRAGON, C. E. C.; MONDRAGON, A. E. C.; MILlER, R. Modularity, open architecture and innovation: an automotive perspective. International Journal of Automotive Technology Management, v. 6, n. 3, p. 346-363, 2006. http://dx.doi.org/10.1504/ IJATM.2006.012125

PANDREMENOS, J. et al. Modularity concepts for the automotive industry: a critical review. CIRP Journal of Manufacturing Science and Technology, v. 1, n. 3, p. 148-152, 2009. http://dx.doi.org/10.1016/j. cirpj.2008.09.012

PERSSON, M. The impact of organizational functions on modular structure: experiences from the Volvo car corporation. International Journal of Automotive Technology and Management, v. 4, n. 1, p. 22-39, 2004. http://dx.doi.org/10.1504/1JATM.2004.004394

PERSSON, M.; AHLSTRÖM, P. Managerial issues in modularizing complex products. Technovation, v. 26, p. 1201-1209, 2006. http://dx.doi.org/10.1016/j. technovation.2005.09.020

PILLER, F. T.; WARINGER, D. Modularisierung in der Automobilindustrie - neue Form und Prinzi - pien. Aachen: Shaker Verlag, 1999.

PIRES, S. R. 1. Managerial implications of the modular consortium model in a Brazilian automotive plant. International Journal of Operations \& Production Management, v. 18, n. 3, p. 221-232, 1998.
PIRES, S. R. 1; CARDOZA, G. A study of new supply chain management practices in the Brazilian and Spanish auto industries. International Journal of Automotive Technology and Management, v. 7, n. 1, p. 72-87, 2007. http://dx.doi.org/10.1504/1JATM.2007.013384

PRIETO, E.; CAUCHICK MIGUEL, P. A. Adoção da estratégia modular por empresas do setor automotivo e as implicações relativas à transferência de atividades no desenvolvimento de produto: um estudo de casos múltiplos. Gestão \& Produção, v. 18, n. 2, p. 425-442, 2011. http://dx.doi.org/10.1590/S0104$530 \times 2011000200015$

RACHID, A. et al. Organização do trabalho na cadeia de suprimentos: os casos de uma planta modular e de uma tradicional na indústria automobilística. Produção, v. 16, n. 2, p. 189-202, 2006.

RAMDAS, K. Managing product variety: an integrative review and research directions. Product and Operations Management, v. 12, n. 1, p. 79-102, 2002. http://dx.doi. org/10.1111/j.1937-5956.2003.tb00199.x

ROZENFELD, $\mathrm{H}$. et al. Gestão de Desenvolvimento de Produto: uma referência para a melhoria do processo. São Paulo: Saraiva, 2006.

SACOMANO NETO, M.; PIRES, S. R. 1. Organização da produção, desempenho e inovações na cadeia de suprimentos da indústria automobilística brasileira. Revista de Ciências da Administração, v. 9, n. 19, p. 34-53, 2007.

SAKO, M.; MURRAY, F. Modules in design, production and use: implications for the global automotive industry. In: GERPISA INTERNATIONAL COLLOQUIUM, 2000, Paris. Proceedings... Paris, 2000.

SAKO, M., MURRAY, F. Modular strategies in cars and computers. Financial Times, 1999.

SALERNO, M. S. The characteristics and the role of modularity in the automotive business. International Journal of Automotive Technology and Management, v. 1, n. 1, p. 92-107, 2001. http://dx.doi.org/10.1504/ IJATM.2001.000029

SALERNO, M. S.; CAMARGO, O. S.; LEMOS, M. B. Modularity ten years after: an evaluation of the Brazilian experience. International Journal of Automotive Technology and Management, v. 8, n. 4, p. 373-381, 2008. http://dx.doi. org/10.1504/IJATM.2008.020309

SALERNO, M. S. et al. The importance of locally commanded design for the consolidation of local supply chain: the concept of design headquarters. International Journal of Manufacturing Technology and Management, v. 16, n. 4, p. 361-376, 2009. http://dx.doi.org/10.1504/ IJMTM.2009.023753

SALERNO, M. S. et al. Mapeamento da nova configuração da cadeia automotiva. São Paulo, 2001. Pesquisa de desenvolvimento junto ao BNDES.

SANCHEZ, R. Using modularity to manage the interactions of technical and industrial design. Design Management Journal, v. 2, p. 8-19, 2002.

SANCHEZ, R.; COLLINS, R. P. Competing and learning in modular markets. Long Range Planning, v. 34, n. 6, p. 645-667, 2001. http://dx.doi.org/10.1016/S00246301(01)00099-1

SANCHEZ, R.; MAHONEY, J. T. Modularity, flexibility and knowledge management in product and organizational design. Strategic Management Journal, v. 17, p. 63-76, 1996. 
TAKEISHI, A.; FUJIMOTO, T. Modularisation in the auto industry: interlinked multiple hierarchies of product, production and supplier systems. International Journal of Automotive Technology and Management, v. 1, n. 4, p. 379-396, 2001. http://dx.doi.org/10.1504/ IJATM.2001.000047

ULRICH, K. T. The role of product architecture in the manufacturing firm. Research policy, v. 24, n. 3, p. 419-440, 1995. http://dx.doi.org/10.1016/00487333(94)00775-3

ULRICH, K. T.; EPPINGER, S. D. Product design and development. McGraw-Hill, New York, 2000.

ULRICH, K. T.; TUNG, K. Fundamentals of product modularity. In: ASME WINTER ANNUAL MEETING SYMPOSIUM ON ISSUES IN DESIGN/MANUFACTURING INTEGRATION, 1991, Atlanta. Proceedings... Atlanta: ASME, 1991.

WORREN, N.; MOORE, K.; CARDONA, P. Modularity, strategic flexibility, and firm performance: a study of the home appliance industry. Strategic Management Journal, v. 23, p. 1123-1140, 2002. http://dx.doi.org/10.1002/smj.276
WU, L.; DE MATTA, R.; LOWE, T. J. Updating a modular product: how to set time to market and component quality. IEEE Transactions on Engineering Management, v. 56, n. 2, p. 298-311, 2009. http://dx.doi.org/10.1109/ TEM.2008.2005065

YIN, R. K. Estudo de Caso: planejamento e métodos. 3. ed. Porto Alegre: Bookman, 2005.

\section{Agradecimentos}

Os autores agradecem o apoio recebido pela Fapesp e CNPq e também a dedicação dos referees que contribuíram significativamente para a melhoria do presente artigo. Um dos autores atua no Programa de Pós-graduação da Escola Politécnica da USP e tem, atualmente, vínculo empregatício com o Departamento de Engenharia de Produção e Sistemas da Universidade Federal de Santa Catarina e, portanto, essa instituição também merece agradecimentos.

\title{
An investigation of product design and production in an automotive assembler and engine suppliers that adopt modularity
}

\begin{abstract}
Modularity can be understood as a set of concepts that leads to new product architecture and production organization. This enables the transference of value-added activities throughout the automotive supply chain. $\mathrm{ln}$ this context, the aim of this paper is to investigate modularity adoption in product design and in production by considering a specific situation of the relationship between a commercial vehicle assembler and engine suppliers. In order to fulfil those objectives, a case-based research was employed as the methodological approach. The results presented some influences that occur between product design and production and vice-versa - part of them were expected. This reinforces the confirmatory nature of the empirical data. The modularity concept may be applied to a productive process not necessarily designed in modules, i.e. it is possible to assemble components as from an existing product, resulting in sub-sets in a modular logic. Data also confirmed that the product architecture of a commercial vehicle favours product division in modules. Moreover, the supply chain management is simplified compared to the traditional production system, as part of the responsibilities is transferred to the suppliers.
\end{abstract}

Keywords

Modularity. Automotive industry. Product design. Product development. 